\title{
Two Kinds of Neural Network Fusion of Aero-engine Rotor Vibration Signal Fault Diagnosis
}

\author{
Feng Ding ${ }^{1, a}$, Zhaoyang Wang ${ }^{2, b}$, Fengwei Qin ${ }^{3, c}$ \\ ${ }^{1}$ Xi'an Technological University, NO.2 Xuefu Middle Road, Xi'an, Shaanxi, P.R. China \\ ${ }^{2}$ Xi'an Technological University, NO.2 Xuefu Middle Road, Xi'an, Shaanxi, P.R. China \\ ${ }^{3}$ Xi'an Technological University, NO.2 Xuefu Middle Road, Xi'an, Shaanxi, P.R. China \\ aemail: 136790921@qq.com, bemail: 254743705@qq.com, cemail:13572022495@163.com
}

Keywords: Aero-engine rotor; Vibration signal; BP neural network; Probabilistic neural network.

\begin{abstract}
In order to improve the accuracy of fault diagnosis, this paper puts forward a method of wavelet packet combining neural network fusion. By wavelet packet decomposition and reconstruction of normal and fault vibration signals of aero-engine rotor, the feature vector from the vibration signal can be extracted. Then put the feature vectors which are the input vector of neural network into the BP (Back Propagation) neural network and PNN(Probabilistic Neural Network), and the paper puts forward an algorithm to fuse the results of BP and PNN. It turns out that the method can recognize the fault patterns well and improve the accuracy of diagnosis.
\end{abstract}

\section{Introduction}

Aero engine is the heart of the modern aircraft, it works in the high pressure, high temperature and high load of the harsh environment ${ }^{[1]}$. Its performance has a direct impact on the safety and reliability of aircraft. The rotor is one of the most important part of the whole engine, which is responsible for the compressed air and power output. The reliability of the engine affects the normal operation of the engine directly. The rotor fault diagnosis can find the faults from the rotor vibration signal, and it can ensure the engine operation reliability, safety and maintenance economy ${ }^{[2]}$.

Aero engine is a very complex nonlinear system, and its fault diagnosis is very difficult. With the development of computer technology, the application of artificial intelligence technology such as neural network, expert system and fuzzy logic in fault diagnosis is promoted ${ }^{[3-5]}$.Neural network technology has been widely used in fault diagnosis because of its self-learning, nonlinear pattern recognition, associative memory and strong functional approximation ability ${ }^{[6,7]}$. Wavelet packet analysis has good high frequency and low frequency analysis characteristics, which play an important role in the process of vibration signal processing, especially the signal de-noising is the key problem to be solved. The main basis of wavelet analysis for noise reduction is the tight support of wavelet basis. It can focus on the energy of the signal in a few large wavelet coefficients, and the wavelet coefficients are generally very small. Compared with the traditional method, the wavelet de-noising only needs to know which type of signal is, and then it can be applied to the standard method of de-noising, which is due to the advantages of wavelet analysis, and it is widely used in signal processing ${ }^{[8-12]}$.

In this paper, wavelet packet analysis is used to extract the fault feature of the rotor system of the aero engine, two kinds of network-BP and probabilistic neural networks are used for pattern identification. At the end, the fusion algorithm is used to fuse the results of two kinds of neural networks. It turns out that, two kinds of neural networks can realize the identification and classification of the rotor fault pattern. And the fusion result is better than both of the two neural networks independently. 


\section{Fusion fault diagnosis system}

The system is divided into three parts, as shown in Figure 1.

(1)Signal processing: First reduce noise of signals. And then use wavelet packet to decompose the signals into four layers. Then reconstruct the 16 decomposition coefficients who is a 16 sub signal, and calculate the ratio of the energy value of the 16 sub signals and the energy value of the original signals. Finally it will generate a $16 \mathrm{D}$ vectors, which are the input vectors of the neural network.

(2)Neural network fault diagnosis: PNN only needs to adjust the spread value. Its training and

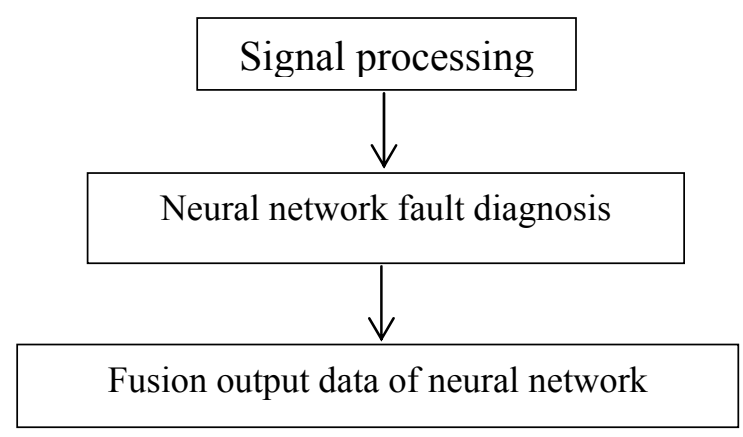

Fig. 1 The fusion fault diagnosis system

testing speed is very fast. Its training time is only slightly larger than the time of data reading, so it meets the requirements of real-time monitoring. BP neural network is one of the most commonly used neural network. Now commonly used neural network is basically BP neural network deformation form. And this network structure is the most suitable for pattern recognition. So fault diagnosis using PNN and BP neural network. The same learning sample is used to train and test the network. The test results of the two kinds of network are used as the input of data fusion.

(3)Fusion output data of neural network: This paper presents an algorithm to fuse the result of the output data of neural network what can make the diagnosis to be more accurate.

\section{Wavelet packet data processing}

According to the main types of aero engine rotor fault(Rotor misalignment, rotor imbalance, rotor and stator friction, bearing loose) $)^{[13]}, 24$ sets of vibration signal(Including the vibration data under normal conditions)were measured for each aero engine fault patterns, then there are 120 sets of vibration data.

\section{The wavelet packet denoising}

This article uses the default threshold and adjusted threshold of the original signal in denoising ${ }^{[14]}$, the results are shown in figure 2.The difference between the default threshold de-noising signal and the original signal is not very obvious. However, the adjusted threshold denoising signal can get the ideal de-noising effect.

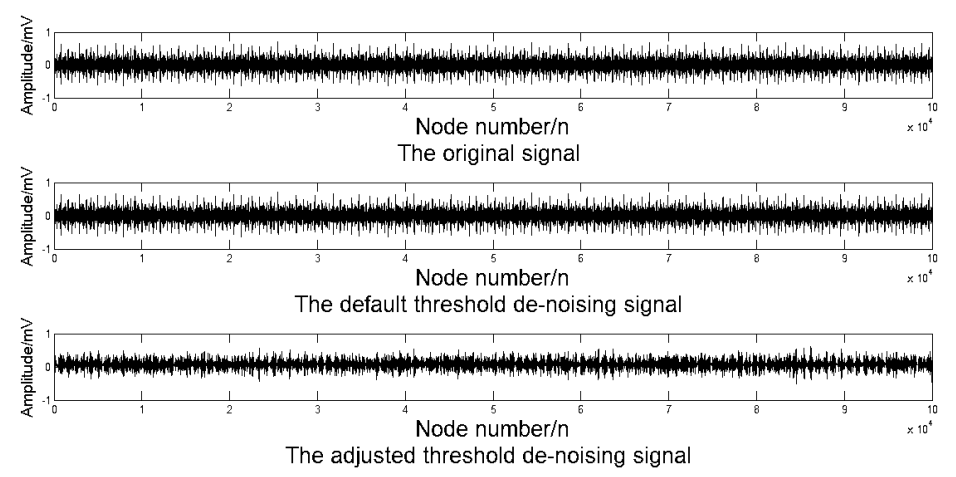

Fig. 2 The result of two kinds of de-noising 


\section{Wavelet packet decomposition and reconstruction of signal}

Using sym6 function according to Shannon entropy principle to decompose de-noised signal into 4 layers in Matlab, extract the 16 sub signals of 4th layer. And then use the wprcoef command to reconstruct these 16 node signals, calculated ratio of the total energy of the original signal value and each sub-band signal energy reconstruction, which are the feature vectors. Figure 3 is the reconstruction of each node in the 16 frequency bands.

\section{The extraction of feature vector}

The signal energy of each frequency band is

$$
E_{N j}=\int\left|S_{N j}(t)\right|^{2}=\left(\sum_{k=1}^{n}\left|x_{j k}\right|^{2}\right)^{\frac{1}{2}} \quad j=1,2, \cdots \cdots, 2^{N}
$$

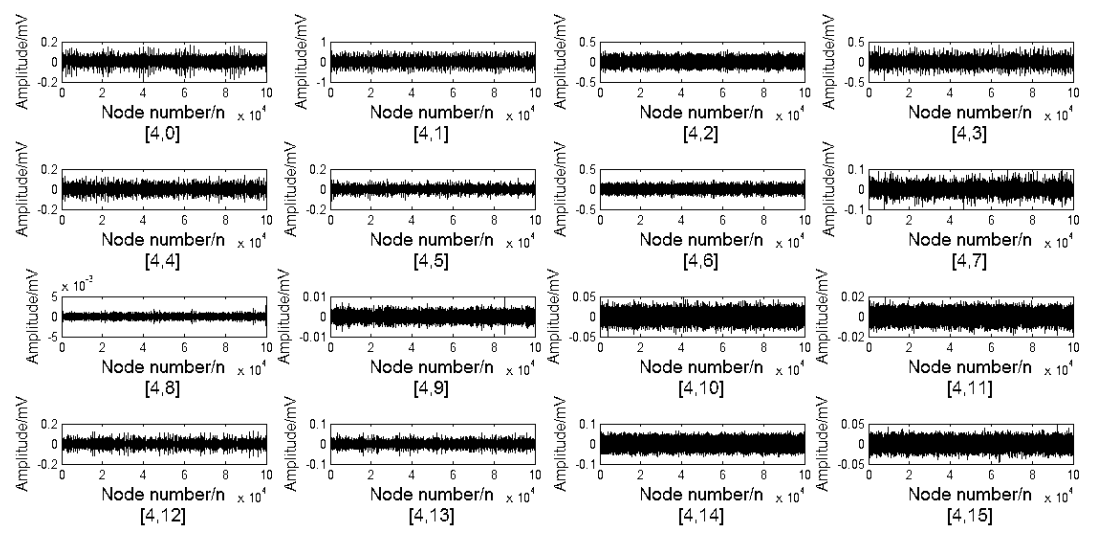

Fig. 3 Reconstruction of each node in the 16 frequency bands

Among them: $S_{N j}$ is the reconstructed signal; $x_{j k}$ is the discrete point amplitude of signal $S_{N j} ; N$ is the number of nodes $S_{N j}$ in signal reconstruction. Total energy is

$$
E=\left(\sum x_{i}^{2}\right)^{\frac{1}{2}} \quad i=1,2, \cdots
$$

$x_{i}$ is the original signal amplitude of each node. The feature vectors of the elements are as follows.

$$
T=\left[E_{N 1}, E_{N 2}, \cdots, E_{N j}\right] \quad j=1,2, \cdots, 2^{N}
$$

$T=\left[E_{N 1}, E_{N 2}, \cdots, E_{N j}\right]$ is usually large numerical, so it needs to do the normalized processing on it, and then get special vector

$$
T^{\prime}=\left(\frac{E_{N 1}}{E}, \frac{E_{N 2}}{E}, \cdots, \frac{E_{N j}}{E}\right)
$$

Type (4) is the final feature vector. The vector is the sample data of neural network fault diagnosis.

\section{Neural network fault diagnosis}

In this paper, The layer number of the BP neural network is 3.And in BP neural network, the node number of the input layer is 16 ,the node number of the hidden layer is 12 , the node number of the output layer is 4.The spread value of the PNN is 0.2 . The failure mode expectation outputs are as in table 1 . 
Table 1 The failure mode expectation output

\begin{tabular}{cc}
\hline Fault mode & Expected output \\
\hline Rotor misalignment & $\left(\begin{array}{llll}1 & 0 & 0 & 0\end{array}\right)$ \\
Rotor unbalance & $\left(\begin{array}{llll}0 & 1 & 0 & 0\end{array}\right)$ \\
Rotor and stator friction & $\left(\begin{array}{llll}0 & 0 & 1 & 0\end{array}\right)$ \\
Bearing loose & $\left(\begin{array}{llll}0 & 0 & 0 & 1\end{array}\right)$ \\
Normal state & $\left(\begin{array}{llll}0 & 0 & 0 & 0\end{array}\right)$ \\
\hline
\end{tabular}

120 sets of vibration signal data in all were collected in this paper, 100 sets of data were used for network training, the remaining 20 sets test were used for test samples. Creating a BP neural network on the basis of the above mentioned methods with newff function, and a PNN function with newgrnn, The results are shown in table 2.

\section{Fusion diagnosis results}

In reality, we always hope the output to be closer to the expected value. For example, we hope that the output of the fault data of rotor misalignment is $(1,0,0,0)$. It is easy to find that the elements of this 4 dimension vector are 0 except for the first one. On the other hand we always hope that the element Table 2 Two neural network diagnosis results

\begin{tabular}{cccccccccccccc}
\hline Number & \multicolumn{1}{c}{ BP neural network diagnosis results } & \multicolumn{7}{c}{ PNN diagnostic results } & \multicolumn{1}{c}{ Expected } \\
\hline 1 & 0.0364 & 0.0073 & 0.015 & 0.0087 & 0.0333 & 0.0000 & 0.0000 & 0.0000 & 0 & 0 & 0 & 0 \\
2 & 0.0264 & 0.0109 & 0.0185 & 0.0052 & 0.0371 & 0.0000 & 0.0000 & 0.0000 & 0 & 0 & 0 & 0 \\
3 & 0.0559 & 0.0117 & 0.0143 & 0.0051 & 0.0349 & 0.0000 & 0.0000 & 0.0000 & 0 & 0 & 0 & 0 \\
4 & 0.0289 & 0.0043 & 0.0178 & 0.0054 & 0.0410 & 0.0000 & 0.0000 & 0.0000 & 0 & 0 & 0 & 0 \\
5 & 0.976 & 0.0145 & 0.0028 & 0.0268 & 0.9455 & 0.0234 & 0.0029 & 0.0000 & 1 & 0 & 0 & 0 \\
6 & 0.9679 & 0.0056 & 0.0014 & 0.0133 & 0.9363 & 0.0163 & 0.0013 & 0.0000 & 1 & 0 & 0 & 0 \\
7 & 0.9571 & 0.0043 & 0.0015 & 0.0176 & 0.9431 & 0.0153 & 0.0015 & 0.0000 & 1 & 0 & 0 & 0 \\
$\mathbf{8}$ & $\mathbf{0 . 4 1 5 5}$ & $\mathbf{0 . 9 7 9 8}$ & $\mathbf{0 . 0 0 3 8}$ & $\mathbf{0 . 0 0 9 7}$ & 0.8326 & 0.0143 & 0.0018 & 0.0000 & 1 & 0 & 0 & 0 \\
9 & 0.0057 & 0.9793 & 0.0088 & 0.0000 & 0.0114 & 0.7511 & 0.2013 & 0.0362 & 0 & 1 & 0 & 0 \\
10 & 0.0064 & 0.9905 & 0.017 & 0.0000 & 0.0117 & 0.716 & 0.2326 & 0.0396 & 0 & 1 & 0 & 0 \\
11 & 0.0102 & 0.9934 & 0.0102 & 0.0000 & 0.0204 & 0.7576 & 0.1956 & 0.0265 & 0 & 1 & 0 & 0 \\
$\mathbf{1 2}$ & $\mathbf{0 . 0 0 7 3}$ & $\mathbf{0 . 0 0 1 8}$ & $\mathbf{0 . 0 2 0 6}$ & $\mathbf{0 . 0 0 0 0}$ & 0.0147 & 0.7676 & 0.1905 & 0.0272 & 0 & 1 & 0 & 0 \\
$\mathbf{1 3}$ & 0.0017 & 0.0029 & 0.9783 & 0.0179 & $\mathbf{0 . 0 0 1 3}$ & $\mathbf{0 . 1 8 9 4}$ & $\mathbf{0 . 6 9 1 3}$ & $\mathbf{0 . 1 1 7 9}$ & 0 & 0 & 0 & 1 & 0 \\
14 & 0.0011 & 0.0042 & 0.9873 & 0.0141 & 0.0008 & 0.1706 & 0.6878 & 0.1409 & 0 & 0 & 1 & 0 \\
$\mathbf{1 5}$ & 0.0000 & 0.0063 & 0.978 & 0.0111 & $\mathbf{0 . 0 0 1 5}$ & $\mathbf{0 . 2 0 3 8}$ & $\mathbf{0 . 6 7 8 2}$ & $\mathbf{0 . 1 1 6 5}$ & 0 & 0 & 1 & 0 \\
16 & 0.0000 & 0.0191 & 0.9465 & 0.009 & 0.0011 & 0.1906 & 0.6912 & 0.1172 & 0 & 0 & 1 & 0 \\
17 & 0.017 & 0.0147 & 0.0233 & 0.973 & 0.0000 & 0.0322 & 0.1274 & 0.8404 & 0 & 0 & 0 & 1 \\
18 & 0.0171 & 0.0104 & 0.0243 & 0.9848 & 0.0000 & 0.0381 & 0.1567 & 0.8052 & 0 & 0 & 0 & 1 \\
19 & 0.0335 & 0.0144 & 0.0254 & 0.983 & 0.0000 & 0.0415 & 0.1524 & 0.8061 & 0 & 0 & 0 & 1 \\
20 & 0.0206 & 0.0144 & 0.0235 & 0.9828 & 0.0000 & 0.0335 & 0.1416 & 0.8249 & 0 & 0 & 0 & 1 \\
\hline
\end{tabular}

the fault mode accounted for the entire vector element ratio is closer to 1 . But in practice we find that although the proportion of elements that represent the failure mode is the largest, the proportion of other elements may also be very large. We call these elements interference terms. The thirteenth output $(0.0013,0.1894,0.6913,0.1179)$ and fifteenth output $(0.0015,0.2038,0.6782,0.1165)$ of the PNN diagnosis can be seen in Table 3 are interference terms. In addition, we compared the eighth set of output 
$(0.4115,0.9789,0.0038,0.0097)$ with its desired output $(1,0,0,0)$ and the twelfth set of output $(0.0073,0.0018,0.0206,0.0000)$ with its expected output $(0,1,0,0)$ of the BP neural network, it's easily found that these two groups of results are obvious diagnostic errors. Using this result as a diagnostic output may reduce the reliability of the diagnosis.

Therefore, this paper puts forward an algorithm to fuse the output of the two kinds of neural networks. It can not only increase the ratio of the element represents the fault mode, but also reduce the ratio of the interference terms. What's more, it combined with the output of two kinds of neural networks, so the accuracy of diagnosis can be improved. The algorithm expression is as follows:

$$
l_{j}^{i}=l b p_{j}^{i} \cdot \frac{l p_{j}^{i} / \sum_{j=1}^{n} l p_{i}^{j}}{\frac{l p_{j}^{i}}{\sum_{j=1}^{n} l p_{j}^{i}}+\frac{l b p_{j}^{i}}{\sum_{j=1}^{n} l b p_{j}^{i}}}+l p_{j}^{i} \cdot \frac{l b p_{j}^{i} / \sum_{j=1}^{n} l b p_{j}^{i}}{\frac{l b p_{j}^{i}}{\sum_{j=1}^{n} l b p_{j}^{i}}+\frac{l p_{j}^{i}}{\sum_{j=1}^{n} l p_{j}^{i}}}
$$

$$
j=1,2, \cdots, n
$$

In this algorithm, $l_{j}^{i}$ is the $j$ th element of $i$ th vector of final output. $l b p_{j}^{i}$ is $j$ th element of $i$ th vector of BP neural network diagnostic output. $I p_{j}^{i}$ is is the $j$ th element of $i$ th vector of PNN diagnostic output.

By fusing the two results of diagnosis of two diagnostic system ,because the real fault component values will dominate in the original vector, it can still keep the larger advantage after fusion; However by inverse proportion complementary, the size and the proportion of disturbance fault component values will be greatly reduced. So this algorithm can make the two complementary advantages. In another hand, it is easy to judge the failure categories more clearly. Table 3 shows the two neural network diagnosis results output after fusion.

\begin{tabular}{|c|c|c|c|c|c|c|c|c|c|c|c|}
\hline No. & & The fus & on results & & Expect & No. & & The fusio & on results & & Expect \\
\hline 1 & 0.0348 & 0.0000 & 000 & 0.0000 & 0000 & 11 & 0.0153 & 0.8220 & 0.0277 & 0.0000 & 0100 \\
\hline 2 & 0.0317 & 0.0000 & 0.0000 & 0.0000 & 0000 & 12 & 0.0110 & 0.8302 & 0.0509 & 0.0000 & 0100 \\
\hline 3 & 0.0454 & 0.0000 & 0.0000 & 0.0000 & 0000 & 13 & 0.0015 & 0.0031 & 0.8291 & 0.0283 & 010 \\
\hline 4 & 0.0349 & 0.0000 & 0.0000 & 0.0000 & 0000 & 14 & 0.0000 & 0.0049 & 0.8343 & 0.0227 & 00010 \\
\hline 5 & 0.9607 & 0.0071 & 0.0028 & 0.0000 & 1000 & 15 & 0.0000 & 0.0062 & 0.8878 & 0.0156 & 0010 \\
\hline 6 & 0.9521 & 0.0153 & 0.0013 & 0.0000 & 1000 & 16 & 0.0000 & 0.0092 & 0.8637 & 0.0128 & 0010 \\
\hline 7 & 0.9501 & 0.0081 & 0.0015 & 0.0000 & 1000 & 17 & 0.0000 & 0.0216 & 0.0314 & 0.9451 & $\begin{array}{llll}0 & 0 & 0 & 1\end{array}$ \\
\hline 8 & 0.7145 & 0.0023 & 0.0064 & 0.0052 & 1000 & 18 & 0.0000 & 0.0183 & 0.0332 & 0.9454 & $\begin{array}{llll}0 & 0 & 0 & 1\end{array}$ \\
\hline 9 & 0.624 & 0.008 & 0.0021 & 0.0000 & 0100 & 19 & 0.0000 & 0.0135 & 0.0346 & 0.9468 & $\begin{array}{llll}0 & 0 & 0 & 1\end{array}$ \\
\hline 10 & 0.009 & 0.7912 & 0.0000 & 0.0000 & 0100 & 20 & 0.0000 & 0.0173 & 0.032 & 0.9497 & $\begin{array}{llll}0 & 0 & 0 & 1\end{array}$ \\
\hline
\end{tabular}

Table 3 The fusion results

Figure 4 shows the percentage of the largest element of the each line of PNN output and the result of the fusion. Because the main elements of the first four elements of the fusion results are 0 , so eliminating the first four lines. It can be seen from figure 4, that fusion results improve the PNN diagnostic output of each line of data in the proportion of the largest element. Comparison of table 2 and table 3 can be found the fusion results are also corrected eighth and twelfth output errors of BP neural network .Namely, the results of fusion improve the accuracy of fault diagnosis. Although the BP neural network has diagnostic error terms, the largest element in the output results of the BP neural network is originally very large. So the contrast of the BP neural network is omitted in figure 3. 


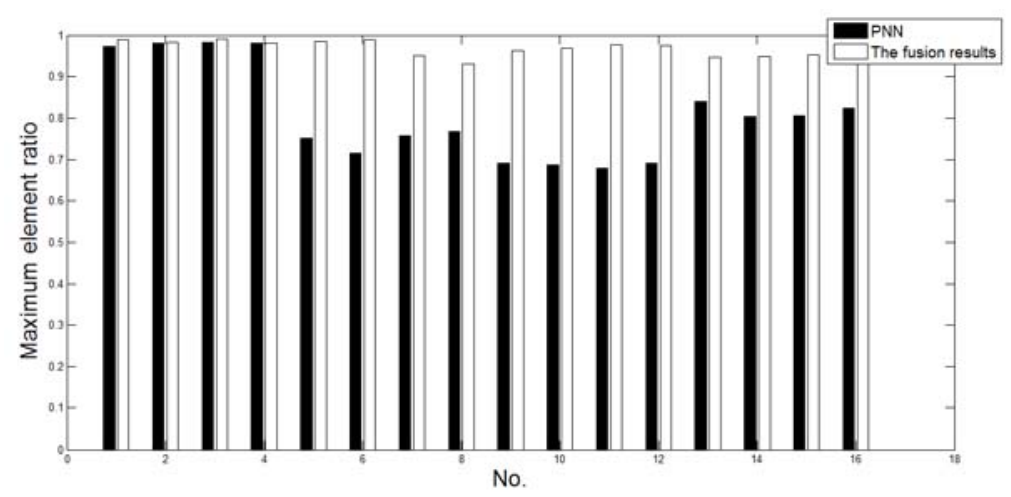

Fig. 4 Comparison of the percentage of maximum elements

\section{Conclusion}

In this paper, the combination of fault diagnosis and diagnostic output information fusion algorithm based on wavelet packet neural network is proposed firstly. Secondly BP neural network and probabilistic neural network are used to identify the fault pattern. Finally fuse the diagnostic output information of BP neural network and PNN, and using the chart and table compares the results as a diagnostic output different before and after fusion. Through calculation of 20 sets of data contrast, the result is visible that the BP neural network diagnosis accuracy rate reached 90\%, and PNN diagnosis results are roughly correct, but for each set of diagnosis of PNN output, proportion of maximal element is low. By comparing, it's can be found that the fusion result not only correct the error of BP neural network diagnosis, but also improve the PNN diagnosis in the proportion of maximal element of each group. On the other hand, the fusion result is better than one of these two neural networks independently to make diagnosis of the output.

\section{Acknowledgment}

This research was financially supported by the National Science Foundation of China under grant 51275374 and the Fund Project of Science \& Technology on Reliability \& Environmental Engineering Key Laboratory, as well as the Scientific Research Program Funded by Shanxi Provincial Education Department (Program No. 11JK0860).

\section{References}

[1]LIU Daxiang,CHEN Guang.Aero Engine: The heart of an airplane[M].Beijing:Aviation Industry Press,2003.

[2]SUN Chuang,HE Zhengjia,ZHANG Zhousuo,CHEN Xuefeng,CAO Hongrui,NING Xueyu,ZOU Limin.Operating Reliability Assessment for Aero-engine Based on Condition Monitoring Information[J].Journal of Mechanical Engineering,2013, 49(6): 30-37.

[3] Lu P J, Zhang M C, H su T C.An evaluation of engine faults diagnostics using artificial neural networks[J].Journal of Engineering for Gas Turbines and Power,2001,123,(2): 340-360.

[4] ZHOU Rusheng,JIAO Zongxia,WANG Shaoping,ZHAO Yan.Fault Diagnosis Expert System for Hydyaulic System of Missile Launcher[J].Journal of aviation,2008, 29(1): 197-203.

[5]SHAN Xiaoming,SONG Yunfeng,HUANG Jinquan,CHOU Xiaojie,LU Feng.Condition Monitoring of Aero-engine based on Neural Network and Fuzzy Logic[J].Journal of Aerospace Power,2009, 24(10): 2356-2361.

[6] QIAN Zhengwen,CHENG Li,ZHAO Bingbing,LI Yingho-ng.Assessment research on Damage Degree of Blade in Aero-engine based on Back-propagation Artificial Neural Networks[J].Journal 
of Aerospace Power,2011, 26(4): 794-799.

[7]ZHAI Xusheng,HU Jinhai,XIE Shousheng,LIU Jia,LI Qiang.Diagnosis of Aero-engine with Early Vibration Fault Symptom using DSmT[J].Journal of Aerospace Power,2011, 26(4): 794-799.

[8]PENG Zhike,LU Wenxiu,ZHU fulei.A new method of vibration signal de-noising based on Wavelet Transform[J].Journal of Mechanical Engineering,2006, 42(4): 18-22.

[9] YANG Shaopu,Zhao Zhihong.Improved Wavelet Denoising Using Neighboring Coefficients and Its Application to Mechinery Fault Diagnosis[J].Journal of Mechanical Engineering,2006, 42(4): $18-22$.

[10]Tang Wei, Shi Zhong-Ke.Wavelet Denoising of Flutter Testing Data for Improvement of Parame-ter Identification[J].Chinese Journal of Aeronautics,2005 18(1): 72-77.

[11] Altmamm J, Mathew J.Multi-band pass autoregressive demodulation for rolling element bearing fault diagnosis[J]. Mechanical System and Signal Processing,2001, 15(5): 963-977.

[12]WANG Fengtao,CHEN Shouhai,YAN Dawen,WANG Lei,ZHU Hong,LIU Enlong.Noise Reduction Based on Dual Tree Complex Wavelet Transformunfolding and Its Application in Fault Diagnosis[J].Journal of Mechanical Engineering,2014, 50(21): 159-163.

[13] AI Yanyan,FEI Chengwei,WANG Zhi.Technique for diagnosing fault of rotor vibration based on improved fuzzy SVM[J].Journal of Aerospace Power,2011,26(5):1118-1123.

[14] WANG Chengdong,ZHU Yongsheng,ZHANG Youyun.Study on vibration signal de-noising method of rotor[J],Vibration, testing and diagnosis,2003, 23(4): 252-255. 